\title{
Evidencia científica como insumo para estrategias sanitarias: ¿un desafío factible o una validación local que susurra a oídos sordos?
}

\author{
Dr. Federico Ferrando
}

Los organismos y las personas que gestionan el Sistema Nacional Integrado de Salud (SNIS) son responsables de definir los problemas prioritarios de la salud y luego ejecutar una serie de acciones de largo aliento que trascienden a los programas de los sucesivos gobiernos.

La ley número 18.211, promulgada el 5 de diciembre de 2007, establece que "lograr el aprovechamiento racional de los recursos humanos, materiales, financieros y de la capacidad sanitaria instalada y a instalarse" es un objetivo principal del SNIS. Naturalmente, el esfuerzo de planificar, ejecutar y monitorizar esta asignación racional de recursos en salud es aun mayor cuando existen inequidades en el acceso a los servicios, menor aporte de fondos o ineficacia en la gestión de los procesos.

Pero pasar del discurso estratégico a las acciones racionales suele ser un camino largo y plagado de obstáculos ${ }^{(1)}$ cuando no un campo minado de dilemas y problemas éticos ${ }^{(2)} \mathrm{o}$ un espejo del fracaso en algunas políticas sanitarias ${ }^{(3)}$.

Las estrategias que impactan sobre la morbimortalidad de las enfermedades cardiovasculares (ECV), principalmente de la cardiopatía isquémica (CI), ejemplifican esta dificultad.

Como primera causa de muerte a nivel mun$\operatorname{dial}^{(4)}$, regional(5) y nacional(6), las ECV constituyen un problema de salud prioritario para varios países latinoamericanos, entre ellos, el nuestro ${ }^{(7)}$. Si a este liderazgo epidemiológico se agregan la complejidad de su abordaje clínico individual y el crecimiento progresivo del arsenal diagnóstico-terapéutico se comprende por qué siguen exigiendo enormes esfuerzos técnicos y económicos ${ }^{(8)}$.

Si bien la mortalidad por enfermedad coronaria ha experimentado un descenso sostenido en Uruguay, pasando de 313 a 252 muertes por 100.000 habitantes entre 2005 y $2014^{(6,9)}$, se desconoce si el aumento progresivo del gasto local en salud(10) (figura 1) se condice con mejoras de la calidad asistencial en los pacientes con CI y otras patologías. Esta es una debilidad mayor del SNIS que viene siendo señalada por las gremiales médicas y por los usuarios sin una respuesta acorde de la rectoría del sistema. En efecto, no se dispone de un plan nacional de acción debidamente consensuado que aborde integralmente el problema de la CI (un "mojón cero" en el camino), ni se realiza un monitoreo sistemático de calidad asistencial a través de indicadores estructurales, de proceso y de resultados (figura 2). Como excepciones cabe mencionar el análisis de morbimortalidad e ingresos-egresos (indicadores de resultado) ${ }^{(9)}$ y los datos desagregados de algunos programas gestionados por la Comisión Honoraria de Salud Cardiovascular (www.cardiosalud.org). En 2016 se reactivó el abordaje de la reperfusión en el infarto agudo de miocardio (IAM). Un grupo de trabajo, que integra la propia Sociedad Uruguaya de Cardiología (SUC), junto a otros actores en salud, formuló un nuevo programa denominado REPIAM (en referencia al demorado PIAM), que se inició con la capacitación de los profesionales que participan en el abordaje clínico inicial y el tratamiento precoz del IAM. Este protocolo pretende unificar criterios para que los pacientes accedan a la reperfusión miocárdica mediante angioplastia primaria o fibrinolíticos en el menor tiempo posible.

Como institución que nuclea a los profesionales del área cardiológica, consideramos que la SUC tiene un compromiso ineludible que consiste en analizar críticamente las estrategias sanitarias para el combate de las ECV a nivel nacional y en buscar los caminos para incidir proactivamente en las mismas. Para quienes tienen la responsabilidad de definir estas estrategias debería ser primordial contar con información rigurosa de primera mano obtenida mediante investigaciones locales y no solo con base en recomendaciones internacionales.

Crear y fortalecer lazos entre la academia (quienes investigan) y la política (quienes deciden) puede contribuir a que los esfuerzos del sistema impacten 


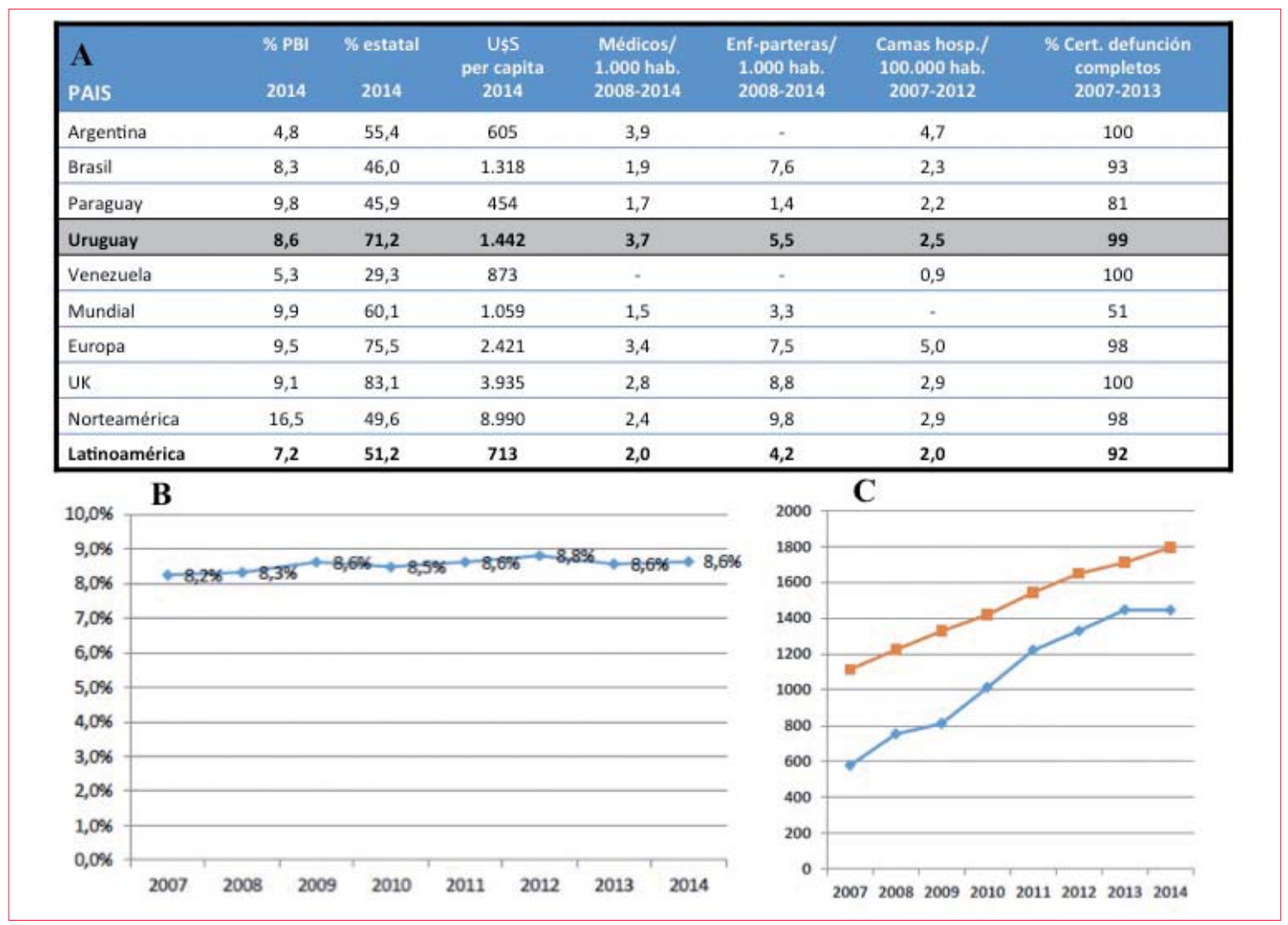

Figura 1. Descriptores del gasto en salud.

A. Descriptores del gasto en salud en diversos países y continentes. Los datos de Uruguay se resaltan en gris ${ }^{(8)}$. B. Evolución del gasto como porcentaje del PBI a nivel mundial en el período 1995-2014(10). Si se extrapolan estos porcentajes al PBI actual, puede estimarse un gasto en Salud Pública mayor a 3.300 millones de dólares en 2016. C. Gasto en salud per cápita en Uruguay (en dólares) desde 2004 a 2015 (línea roja) y per cápita en términos de paridad del poder adquisitivo (línea azul)(10).

más eficazmente en la asistencia de la población ${ }^{(1,12,13)}$. En la práctica, el desarrollo de estrategias sanitarias basadas en información empírica no es solo un problema técnico de intercambio de información o traslación de conocimientos, sino también un desafío político: “...las mejoras en los sistemas de información serán insuficientes si no hay cambios en la manera en que los efectores usan esta información..."(13,14). La Revista Uruguaya de Cardiología (RUC) es herramienta fundamental en este desafío.

En los números 2 y 3 de la RUC se incluyen cinco investigaciones originales que abordan la complejidad local de la CI y proveen datos válidos sobre la calidad de los procesos asistenciales. Estas experiencias se refieren a diferentes presentaciones de la enfermedad como el IAM, la enfermedad coronaria estable y la evolución posoperatoria tras cirugía de revascularización.

En el primero de ellos, el Dr. Torres caracteriza el uso de fibrinolíticos (FBL) bajo cobertura del Fondo Nacional de Recursos en 841 pacientes con infarto de miocardio con elevación de ST (IAMCEST) asistidos entre 2011 y 2014, confir- mando la subutilización de estos agentes en el interior del país y una franca inequidad en el acceso al tratamiento entre los subsistemas público y privado. Los resultados evidencian demoras importantes en el tiempo dolor-aguja y señalan que solo la mitad de los pacientes con infarto en curso recibió FBL en los primeros 45 minutos tras el primer contacto médico. Estos datos, que son alarmantes, obligan a revisar y mejorar los procesos asistenciales en el acceso inmediato a los FBL, en especial intentando acortar el tiempo desde el inicio del dolor al primer contacto médico. Aunque el análisis presenta ciertas limitaciones, como la obtención de datos a partir de formularios que son perfectibles y la carencia de un análisis multivariado que aportaría más consistencia a los resultados, se trata de un relevamiento extenso y exhaustivo muy valioso, que puede servir como insumo para el REPIAM y también como generador de hipótesis para futuras investigaciones.

En el segundo original, el Dr. Conti y colaboradores analizan la tasa de angiografías coronarias sin lesiones epicárdicas ("en blanco") de indicación electiva en un centro público con facilidades educa- 


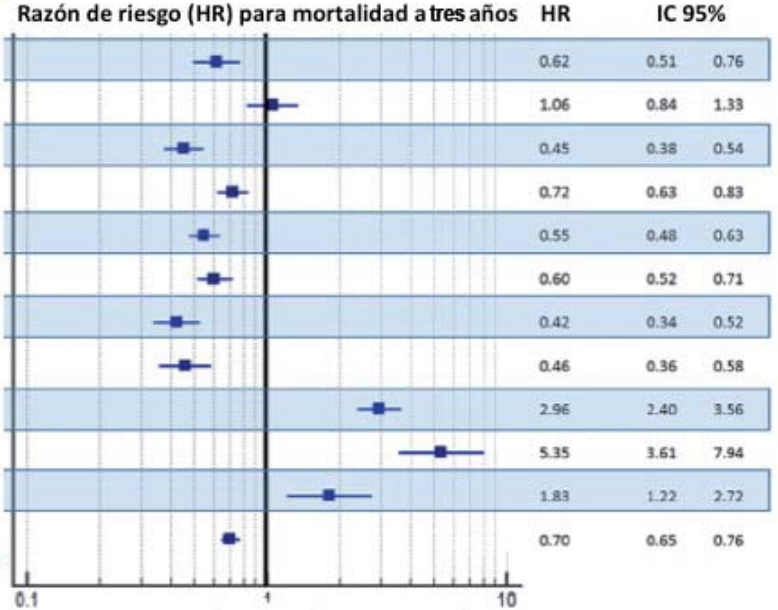

Figura 2. Indicadores de calidad asistencial en los síndromes coronarios agudos.

Se presentan algunos indicadores utilizados para evaluar la calidad asistencial en los síndromes coronarios agudos y su correlato pronóstico. Los indicadores de calidad que se asociaron con la sobrevida a tres años mediante regresión proporcional de Cox están ajustados por características clínicas basales, tipo de infarto, deciles del score GRACE, carga asistencial del centro, disponibilidad de laboratorios de hemodinamia onsite y período (2005-2010) (modificado de ref. 15).

tivas. Sus hallazgos concuerdan con lo reportado en las grandes series internacionales y se ajustan a los estándares de calidad de los centros de hemodinamia, a la vez que sugieren una importante prevalencia de disfunción coronaria microvascular que requiere confirmación en futuras investigaciones.

La Dra. Barranco y colaboradores procesan nuevos datos de una casuística local de angioplastias primarias publicada en $2014^{(16)}$. En este subestudio, los autores encuentran diferencias significativas en la accesibilidad y los resultados de la angioplastia primaria entre hombres y mujeres con IAMCEST. Estos hallazgos transforman una mirada miope de meras diferencias clínico-evolutivas asociadas a caracteres biológicos en un enfoque de potenciales inequidades ligadas al género durante el acceso al intervencionismo percutáneo.

La evolución neurológica en el posoperatorio de la cirugía de bypass coronario es abordada por la Dra. Dotti y colaboradores a través de la pesquisa de signos sugestivos de delirio en un centro privado con alta carga quirúrgica. Mediante un diseño prospectivo, los autores encuentran una incidencia acumulada de delirio elevada $(51,6 \%$ al despertar y 41,9\% durante la internación tras la extubación) y una fuerte asociación de esta entidad con una mayor estadía en terapia intensiva.

Finalmente, la Dra. Sapriza y colaboradores exponen los resultados del trabajo que mereció el galardón a mejor investigación original en el XXXI Congreso Uruguayo de Cardiología. La experiencia demuestra el impacto positivo de una estrategia de traslado directo en el IAMCEST, desde domicilio a un centro de hemodinamia, disminuyendo el tiempo isquémico total a expensas de su componente primer contacto médico-balón y mejorando los índices de contractilidad ventricular izquierda a corto plazo. Aunque se trata de una estrategia unicéntrica desarrollada en el área metropolitana, sus resultados podrían estimular a una replicación en otros ámbitos, como en el interior del país, considerando la puesta en marcha del nuevo centro de hemodinamia de la regional norte, o en el subsector público.

Además de estos originales se incluyeron editoriales, artículos de opinión, revisiones y cartas científicas, conjugando contenido riguroso y fácil lectura. Mientras algunos lectores estarán redactando un borrador de manuscrito para enviarnos, esperamos que todos disfruten los números 2 y 3 de la revista.

\section{Bibliografía}

1. Martin D, Singer P. A strategy to improve priority setting in health care institutions. Health Care Anal 2003; 11(1):59-68.

2. Brock DW, Wikler D. Ethical issues in resource allocation, research, and new product development. En: Jamison DT, Breman JG, Measham AR, Alleyne G, Claeson M, Evans DB, et al, eds. Source disease control priorities in developing countries. 2 ed. Washington, DC: World Bank, 2006.

3. Hipgrave DB, Alderman KB, Anderson I, Soto EJ. Health sector priority setting at meso-level in lower and middle income countries: lessons learned, 
available options and suggested steps. Soc Sci Med $2014 ; 102: 190-200$.

4. Lloyd-Jones D, Adams RJ, Brown TM, Carnethon M, Dai S, De Simone G, et al. Heart disease and stroke statistics - 2010 update: a report from the American Heart Association. Circulation 2010; 121(7):e46-e215.

5. Rodríguez T, Malvezzi M, Chatenoud L, Bosetti C, Levi F, Negri E, et al. Trends in mortality from coronary heart and cerebrovascular diseases in the Americas: 1970-2000. Heart 2006; 92(4):453-60.

6. Curto S, Prats O, Zelarayan M. Mortalidad por enfermedades cardiovasculares. Uruguay, 2009. Rev Urug Cardiol 2011; 26(3):189-196.

7. Uruguay. Ministerio de Salud Pública. Objetivos sanitarios nacionales 2020. Montevideo. MSP, 2016 .

8. World Bank Group. World development indicators 2015. Washington, DC: World Bank, 2015.

9. Uruguay. Comisión Honoraria para la Salud Cardiovascular. Área de Epidemiología y Estadística. Morbilidad y mortalidad por enfermedades cardiovasculares en el Uruguay, 2014. Morbilidad de enfermedades cardiovasculares en el Uruguay 2013-2014. Montevideo: CHSC, 2015.

10. Uruguay. Ministerio de Salud Pública. Área Economía de la Salud. Equipo de Cuentas de Salud. Cuentas de salud de Uruguay: cifras preliminares 2011-2014 bajo SHA 2011. Montevideo: MSP, 2016.

11. American Heart Association. American College of Cardiology. Measuring and improving qua- lity of care: a report from the American Heart Association/American College of Cardiology First Scientific Forum on Assessment of Health Care Quality in Cardiovascular Disease and Stroke. Circulation 2000; 101(12):1483-93.

12. Brick A, Nolan A, O'Reilly, Smith S. Resource allocation, financing and sustainability in health care: evidence for the Expert Group on resource allocation and financing in the health sector. Dublin: The Economic and Social Research Institute, 2010.

13. Liverani M, Hawkins B, Parkhurst JO. Political and institutional influences on the use of evidence in public health policy: a systematic review. PLoS One 2013; 8(10):e77404.

14. Humphreys K, Piot P. Scientific evidence alone is not sufficient basis for health policy. BMJ 2012; 344:e1316.

15. Schiele F, Gale CP, Simon T, Fox KAA, Bueno H, Lettino M, et al. Assessment of Quality Indicators for Acute Myocardial Infarction in the FAST-MI (French Registry of Acute ST-Elevation or Non-ST-Elevation Myocardial Infarction) Registries. Circ Cardiovasc Qual Outcomes 2017; 10(6). pii: e003336.

16. Mayol J, Perna A, Albornoz H; Comité de Hemodinamia de la Sociedad Uruguaya de Cardiología. Angioplastia en el infarto de miocardio dentro de las 24 horas de evolución: análisis de la casuística uruguaya 2004-2012. Rev Urug Cardiol 2014; 29(1):6-16.. 